\section{Determination of synthetic lethal interactions to provide therapeutic direction to end aggressive prostate}

\section{cancer}

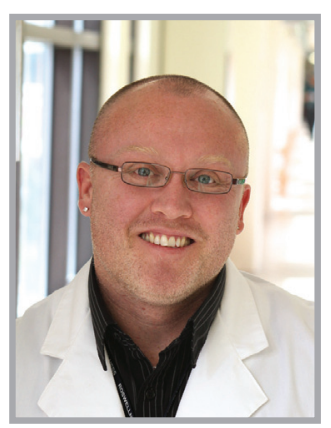

\author{
"While recent advances in whole-genome analysis \\ have provided a more clear vision of the \\ genomic/epigenomic prostate cancer landscape, \\ our complete understanding of critical synergistic \\ interactions that drive aggressive prostate cancer \\ phenotypes and their separation from casual \\ passenger events is only starting to emerge."
}

Leigh Ellis*

Emergence of aggressive prostate cancer (PCa) phenotypes resistant to current available therapies is a significant clinical problem, and novel therapeutic strategies are urgently required. Androgen receptor (AR) action is a key mediator underlying development and progression of PCa. For this reason, androgen deprivation therapy has been a primary standard of care for men for over 70 years with symptomatic, advanced PCa [1]. Unfortunately, ensuing resistance to therapy is inevitable, and men will eventually develop castrationresistant PCa (CRPC). Further delineation of underlying mechanisms driving CRPC led to the finding that CRPC is not androgen independent, but continues to rely on androgen and AR activity. This knowledge has resulted in the development of second-generation agents which either target the AR or the synthesis of extragonadal androgens [2]. More specifically, the agents abiraterone acetate and enzalutamide received US FDA approval based on clinical trial data displaying modest survival benefit compared with placebo in men with CRPC [3]. Despite advances with such therapies, approximately $20-40 \%$ of CRPC patients have no response (de novo resistance), or resistance to these agents develops rapidly in those patients who initially respond (acquired resistance) [4]. Reports now implicate underlying mechanisms of resistance as increased expression of truncated ligand-independent AR variants or castration-resistant neuroendocrine $\mathrm{PCa}$, which are negative for AR expression [5,6]. One overlapping feature independent of phenotype is the high replicative/proliferative nature of these tumors. This insight highlights DNA replication forks and associated machinery as promising targets to treat these aggressive $\mathrm{PCa}$ phenotypes. Another important point to improve the clinical management of aggressive $\mathrm{PCa}$ is the extension of our knowledge of gene/gene or gene/pathway cross talk that identifies bona fide synthetic lethal interactions that drive aggressive disease evolution and apply this knowledge to novel therapeutic applications.

An underutilized approach to discover gene interactions in PCa is cross-species analysis. This analysis involves the use of

*Genitourinary Program, Department of Pharmacology \& Therapeutics, Roswell Park Cancer Institute, 665 Carlton Street, Buffalo, NY 14263, USA; Leigh.Ellis@roswellpark.org

Future
Medicine
part of

\section{KEYWORDS}

- aggressive prostate cancer

- epigenetics • ezh2 • metastasis

- precision medicine $\bullet$ targeted

therapy • topoisomerase

“Emergence of aggressive prostate cancer phenotypes resistant to current available therapies is a significant clinical problem, and novel therapeutic strategies are urgently required." 


\section{"Overall, significant increases of therapeutic options for patients with advanced prostate cancer have been achieved; however, these options only subdue disease progression for a relative short period of time."}

animal models to identify gene synergies, validate their functional relevance and then relate this knowledge to human PCa. An example of this included a recent genome-wide analysis of multiple interactomes between human and mouse $\mathrm{PCa}$, using expression profiles of human PCa samples and genetically engineered mouse models, respectively. Cross-species analysis identified a FOXM1 and CENPF functional gene synergy and coexpression of FOXM1 and CENPF mRNA was a robust prognostic indicator of poor survival and metastasis [7]. Further, gene synergies have been investigated for many years in the context for synthetic lethal interactions with hope of translating these findings to clinical therapeutic applications. Recent success of PARP inhibitors is an example of this, one of the first targeted therapies to be identified with synthetic lethal interaction with mutated genes involved in DNA repair [8]. Recently, it was demonstrated that inhibition of the histone methyltransferase Ezh2 acts in a synthetic lethal manner in ARIDIA-mutated ovarian cancer cells [9].

DNA replication forks represent potential landmines throughout the cancer genome and have been the target of many chemotherapy agents. Agents such as etoposide which target topoisomerase II enzymes such as Top2a and mediate DNA replication stalling which result in accumulation of DNA double-strand breaks (DSB) and tumor cell death have been widely used to treat multiple cancer types. Moreover, etoposide has been trialed as a therapeutic strategy in CRPC [10], though current clinical data do not currently provide convincing evidence toward the most beneficial use of etoposide in CRPC patients [10,11]. Also, etoposide toxicities frequently make this agent difficult for clinical use. Optimally, if one could harness the potent antitumor effect of these agents with minimal toxicity, therapies such as etoposide are potent options for these highly proliferative aggressive PCa. With this, using low-dose etoposide in combination to achieve tumor exclusive synergistic cytotoxicity by inducing DNA replication stalling and cell death would provide rapid impact to the treatment of patients with CRPC exhibiting increased AR variant expression or neuroendocrine PCa. An example of this involved a previous description of an interaction between AR and topoisomerase II beta (Top2b) in PCa cell lines [12]. This discovery brought about the initiation of a clinical trial to test the feasibility of combining androgen cycling (androgen deprivation therapy cycled with androgen supplementation) with etoposide. All patients were reported to demonstrate PSA reductions and therefore provided proof of concept to further evaluate this therapeutic approach in larger trials for men with CRPC [13].

Our recent work using a transplant mouse model of PCa described a significant increase of Top $2 a$ expression in metastatic tumors. Using cross-species analysis we correlated increased Top $2 a$ in human metastatic PCa and identified a novel positive correlation between Top $2 a$ expression and expression of $E z h 2$ [14]. This interaction led to the evaluation of a combinatorial therapy approach with the Top2 poison etoposide and Ezh2 inhibitors, GSK126 and DZNep. Single treatment with each agent did not display significant antitumor activity, but when etoposide and the Ezh2 inhibitors were combined, significant increase of cell death in vitro and therapeutic efficacy in vivo were achieved [14]. Further support of this combinatorial approach was illustrated when non-small-cell lung cancer cell lines harboring either EGFR or BRG1 mutations where treated with the Ezh2 inhibitors; sensitivity to etoposide was significantly enhanced. This combinational effect was specific for these mutations as non-small-cell lung cancer cell lines expressing wild-type $E G F R$ or $B R G 1$ upregulated $B R G 1$ in response to Ezh2 inhibition and increased resistance to etoposide [15]. Overall, these exciting results suggest this therapeutic combinatorial approach can provide a synthetic lethal therapybased modality for patients with aggressive $\mathrm{PCa}$ phenotypes, as well as other cancers.

Both Top2a and Ezh2 are associated with characteristic features of aggressive phenotypes in cancer, including PCa $[16,17]$. Top2a and Ezh2 are implicated in mediating alterations in gene expression, as well as increases in cell proliferation, invasive potential and DNA replication $[16,17]$. Also, Top2a and Ezh2 are shown to localize at DNA replication forks; Top2a evoking transient DSB and resolving DNA topological constraints, and Ezh2 acting as part of a DSB repair mechanism [18,19]. In embryonic stem cells, Top2a targets a subset of promoter regions marked by bivalent histone modifications (poised chromatin), suggesting a synergistic role for Top2a and Ezh2 in priming genes for subsequent activation during cell differentiation [20]. These data would imply that concurrent overexpression of Top2a and Ezh 2 could potentially be involved in PCa cancer stem cell maintenance; 
though recently, a PCa study revealed that Top2a increased expression was associated with a recurrence and metastatic phenotype, rather than the PCa cancer stem cell population [16]. Therefore, Top2a and Ezh2 may synergize to regulate gene expression that increases cellular plasticity providing greater metastatic potential and ability to elude current therapies.

Overall, significant increases of therapeutic options for patients with advanced PCa have been achieved; however, these options only subdue disease progression for a relative short period of time. While recent advances in whole-genome analysis have provided a more clear vision of the genomic/epigenomic PCa landscape, our complete understanding of critical synergistic interactions that drive aggressive $\mathrm{PCa}$ phenotypes and their separation from casual passenger events is only starting to emerge. Our work and others [7], highlight the importance to utilize animal models for cross-species analysis to human PCa to identify and functionally validate relevant synergistic interactions (master regulators) of aggressive PCa phenotypes. The ability to perform such analysis will provide critically needed biomarkers for earlier identification of aggressive disease and therapeutic targets which will provide sustained disease progression for patients with CRPC.

\section{Financial \& competing interests disclosure}

The author has no relevant affliations or financial involvement with any organization or entity with a financial interest in or financial conflict with the subject matter or materials discussed in the manuscript. This includes employment, consultancies, honoraria, stock ownership or options, expert testimony, grants or patents received or pending, or royalties.

No writing assistance was utilized in the production of this manuscript.

\section{References}

Papers of special note have been highlighted as:

- of interest; $\bullet$ of considerable interest

1 Huggins C, Hodges CV. Studies on prostatic cancer I. The effect of castration, of estrogen and androgen injection on serum phosphatases in metastatic carcinoma of the prostate. $C A$ Cancer J. Clin. 22(4), 232-240 (1972).

2 Longo DL. New therapies for castrationresistant prostate cancer. N. Engl. J. Med. 363(5), 479-481 (2010).

3 Ryan CJ, Tindall DJ. Androgen receptor rediscovered: the new biology and targeting the androgen receptor therapeutically. J. Clin. Oncol. 29(27), 3651-3658 (2011).

4 Antonarakis ES, Lu C, Wang H et al. AR-V7 and resistance to enzalutamide and abiraterone in prostate cancer. $N$. Engl. J. Med. 371(11), 1028-1038 (2014).

-• Leading clinical study that provides evidence of increased androgen receptor variants expression with de novo resistance to leading treatments for aggressive prostate cancer by analysis of circulating tumor cells.

5 Dehm SM, Schmidt LJ, Heemers HV, Vessella RL, Tindall DJ. Splicing of a novel androgen receptor exon generates a constitutively active androgen receptor that mediates prostate cancer therapy resistance. Cancer Res. 68(13), 5469-5477 (2008).

6 Aggarwal R, Zhang T, Small EJ, Armstrong AJ. Neuroendocrine prostate cancer: subtypes, biology, and clinical outcomes. J. Natl Compr. Canc. Netw. 12(5), 719-726 (2014).
- Comprehensive summary of underlying molecular mechanisms driving neuroendocrine prostate cancer.

7 Aytes A, Mitrofanova A, Lefebvre C et al. Cross-species regulatory network analysis identifies a synergistic interaction between FOXM1 and CENPF that drives prostate cancer malignancy. Cancer Cell 25(5), 638-651 (2014).

- Leading study displaying the power of cross-species analysis to identify and functionally validate gene synergies driving aggressive prostate cancer.

8 Chan DA, Giaccia AJ. Harnessing synthetic lethal interactions in anticancer drug discovery. Nat. Rev. Drug Discov. 10(5), 351-364 (2011).

9 Bitler BG, Aird KM, Garipov A et al. Synthetic lethality by targeting EZH2 methyltransferase activity in ARID1Amutated cancers. Nat. Med. 21(3), 231-238 (2015).

-• First study to demonstrate synthetic lethal interaction involving the histone methyltransferase, EZH2.

10 Pienta KJ, Lehr JE. Inhibition of prostate cancer growth by estramustine and etoposide: evidence for interaction at the nuclear matrix. J. Urol. 149(6), 1622-1625 (1993).

11 Millikan R, Logothetis C. Update of the NCCN guidelines for treatment of prostate cancer. Oncology 11(11A), 180-193 (1997).
12 Haffner MC, De Marzo AM, Meeker AK, Nelson WG, Yegnasubramanian S. Transcription-induced DNA double strand breaks: both oncogenic force and potential therapeutic target? Clin. Can. Res. 17(12), 3858-3864 (2011).

13 Schweizer MT, Antonarakis ES, Wang $\mathrm{H}$ et al. Effect of bipolar androgen therapy for asymptomatic men with castration-resistant prostate cancer: results from a pilot clinical study. Sci. Trans. Med. 7(269), 269ra262 (2015).

- Leading clinical study highlighting the potential for bipolar androgen therapy and etoposide in patients with aggressive prostate cancer.

14 Kirk JS, Schaarschuch K, Dalimov Z et al. Top2a identifies and provides epigenetic rationale for novel combination therapeutic strategies for aggressive prostate cancer. Oncotarget 6(5), 3136-3146 (2014).

-• First study to demonstrate gene synergy between Top2a and Ezh2, and the potential for combination therapy in patients with aggressive prostate cancer.

15 Fillmore CM, Xu C, Desai PT et al. EZH2 inhibition sensitizes $B R G 1$ and $E G F R$ mutant lung tumours to TopoII inhibitors. Nature doi:10.1038/nature14122 (2015) (Epub ahead of print).

- Demonstrates the potential synergy between Ezh2 and Top2 inhibition in non-small-cell lung cancer.

16 Li X, Liu Y, Chen Wet al. TOP2A $\mathrm{A}^{\text {high }}$ is the phenotype of recurrence and metastasis 
EDITORIAL Ellis

whereas TOP2 ${ }^{\text {neg }}$ cells represent cancer stem cells in prostate cancer. Oncotarget 5(19), 9498-9513 (2014).

17 Yang YA, Yu J. EZH2, an epigenetic driver of prostate cancer. Protein Cell 4(5), 331-341 (2013).
18 T, Sun Y, Ji P, Kopetz S, Zhang W. Topoisomerase II $\alpha$ in chromosome instability and personalized cancer therapy. Oncogene doi:10.1038/onc.2014.332 (2014) (Epub ahead of print).

19 Piunti A, Rossi A, Cerutti A et al. Polycomb proteins control proliferation and transformation independently of cell cycle checkpoints by regulating DNA replication. Nat. Comm. 5, 3649 (2014).

20 Thakurela S, Garding A, Jung J, Schubeler D, Burger L, Tiwari VK. Gene regulation and priming by topoisomerase II $\alpha$ in embryonic stem cells. Nat. Comm. 4, 2478 (2013). 